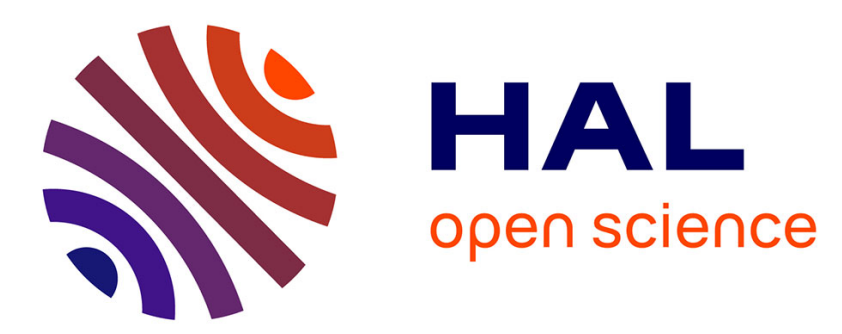

\title{
A substrate sensor chip to assay the enzymatic activity of Botulinum neurotoxin A
}

Christian Lévêque, Géraldine Ferracci, Yves Maulet, Chloé Grand-Masson, Marie-Pierre Blanchard, Michael Seagar, Oussama El Far

\section{To cite this version:}

Christian Lévêque, Géraldine Ferracci, Yves Maulet, Chloé Grand-Masson, Marie-Pierre Blanchard, et al.. A substrate sensor chip to assay the enzymatic activity of Botulinum neurotoxin A. Biosensors and Bioelectronics, 2013, 49, pp.276-281. 10.1016/j.bios.2013.05.032 . hal-00958814

\section{HAL Id: hal-00958814 https://hal.science/hal-00958814}

Submitted on 13 Mar 2014

HAL is a multi-disciplinary open access archive for the deposit and dissemination of scientific research documents, whether they are published or not. The documents may come from teaching and research institutions in France or abroad, or from public or private research centers.
L'archive ouverte pluridisciplinaire HAL, est destinée au dépôt et à la diffusion de documents scientifiques de niveau recherche, publiés ou non, émanant des établissements d'enseignement et de recherche français ou étrangers, des laboratoires publics ou privés. 


\section{A substrate sensor chip to assay the enzymatic activity of Botulinum neurotoxin A}

Christian Lévêque ${ }^{\mathrm{a}, \mathrm{c},{ }^{*}}$, Géraldine Ferracci ${ }^{\mathrm{b}, \mathrm{c}, \mathrm{\&}}$, Yves Maulet ${ }^{\mathrm{a}, \mathrm{c}, \&}$, Chloé Grand-Masson ${ }^{\mathrm{b}, \mathrm{c}}$, Marie-Pierre Blanchard ${ }^{\mathrm{b}, \mathrm{c}}$, Michael Seagar ${ }^{\mathrm{a}, \mathrm{c}}$, and Oussama El Far ${ }^{\mathrm{a}, \mathrm{c}}$

a. INSERM, UMR_S 1072, 13015 Marseille, France

b. CNRS, UMR 7286, Plate-Forme de Recherche en Neurosciences PFRN, 13015 Marseille, France

c. Aix-Marseille Université, 13015 Marseille, France

\&. The authors contributed equally to this work

*Address correspondence to this author at: INSERM 1072, Faculté de médecine secteur nord, Aix-Marseille Université CS80011 Bd Pierre Dramard 13344 Marseille Cedex 15 France. Fax +33-491090506; e-mail christian.leveque@univ-amu.fr 


\section{ABSTRACT}

Botulinum neurotoxin $A$ (BoNT/A) induces muscle paralysis by enzymatically cleaving the presynaptic SNARE protein SNAP-25, which results in lasting inhibition of acetylcholine release at the neuromuscular junction. $A$ rapid and sensitive in vitro assay for BoNT/A is required to replace the mouse lethality assay $\left(L D_{50}\right)$ in current use. We have developed a fully automated sensor to assay the endoprotease activity of BoNT/A. We produced monoclonal antibodies (mAbs) that recognize SNAP-25 neo-epitopes specifically generated by BoNT/A action. Recombinant SNAP-25 was coupled to the sensor surface of a surface plasmon resonance (SPR) system and samples containing BoNT/A were injected over the substrate sensor. On-line substrate cleavage was monitored by measuring binding of mAb10F12 to a SNAP-25 neo-epitope. The SNAP25-chip assay was toxin serotype-specific and detected $55 \mathrm{fM}$ BoNT/A $\left(1 \mathrm{LD}_{50} / \mathrm{ml}\right)$ in 5 minutes and $0.4 \mathrm{fM}\left(0.01 \mathrm{LD}_{50} / \mathrm{ml}\right)$ in 5 hours. Time-course and dose-response curves were linear, yielding a limit of quantification of 0.03 $\mathrm{LD}_{50} / \mathrm{ml}$. This label-free method is 100 times more sensitive than the mouse assay, potentially providing rapid read-out of small amounts of toxin for environmental surveillance and the quality control of pharmaceutical preparations.

Nonstandard abbreviations: BoNT, botulinum neurotoxin; SPR, surface plasmon resonance; $R U$, resonance unit; BSA, bovine serum albumin; $L D_{50}$, median lethal dose; Hepes, 4-(2hydroxyethyl)-1-piperazine ethane sulfonic acid; SNAP-25, Synaptosomal-associated protein 25; DTT, Dithiothreitol; HBS, HEPES-buffered saline; LOD, limit of detection; LOQ, limit of quantification.

Key words: botulinum neurotoxin; SNAP-25; surface plasmon resonance; toxin sensor; endoprotease; neo-epitope. 


\section{Introduction}

Neurotoxins from Clostridium botulinum (BoNTs) act at the neuromuscular junction and cause the human disease botulism. BoNTs display endoprotease activity specific for the SNARE proteins that support $\mathrm{Ca}^{2+}$-dependent exocytosis at the nerve terminal. Thus, cleavage of SNAREs by BoNTs inhibits acetylcholine release and compromises muscle contraction. Most cases of botulism occur through ingestion of contaminated food, with the toxin reaching peripheral nerve terminals via the circulation. Although human botulism is relatively rare, the intoxicated patient needs prolonged and extensive clinical support to counteract descending flaccid paralysis, which can lead to death by respiratory failure if untreated.

Seven different BoNT-serotypes are known, but four (BoNT/A, B, E and F) predominate in human botulism. BoNT/A is the most frequent toxin serotype detected in human botulism in the USA (Arnon et al., 2001). It is also the most potent and can persist in an active form in neurons for weeks or months. BoNT/A was the first BoNT to be introduced for clinical use in the 1980s. Since then, its indications have expanded steadily and BoNT/A is currently used therapeutically to inhibit hyper-contraction or hyper-secretion in a large range of human diseases, as well as cosmetically to reduce facial wrinkles. Moreover BoNT/A is a class A agent that constitutes a potential biological weapon (Dhaked et al., 2010).

BoNT/A occurs naturally as a high molecular mass complex of up to $900 \mathrm{kDa}$, composed of the neurotoxin $(150 \mathrm{kDa})$ and a set of non-toxic components of Clostridial origin. The neurotoxin has two polypeptide chains, linked by a single disulfide bond and non-covalent interactions. Each chain has a distinct function. In vivo the heavy chain (100 kDa) vectors the toxin to motoneuron nerve terminals, via recognition of a specific receptor (synaptic vesicle protein 2 or SV2, associated with gangliosides). Binding results in endocytosis of the toxin followed by translocation of the light chain into the cytoplasm, concomitant with disulfide bond reduction. The light chain (50 kDa) possesses zinc-dependent endopeptidase activity, which specifically cleaves 9 amino acids from the C-terminus of the SNARE protein SNAP-25 (synaptosome-associated protein of $25 \mathrm{kDa}$ ). SNAP-25, which is anchored at the cytoplasmic surface of the synaptic plasma membrane, is a key component of the synaptic vesicle fusion machinery (Rossetto et al., 2013).

The standard method, used currently for serological diagnosis of botulism, surveillance and quality control of batches during pharmaceutical production, is the mouse toxicity bioassay, which has a detection limit of $1 \mathrm{LD}_{50} / \mathrm{ml}$ (10-20 pg BoNT/A). However this procedure is 
increasingly considered as unethical and furthermore is relatively slow (24-48 h), as the symptoms of botulism in the mouse can take several days to appear. Thus the mouse assay is poorly adapted to emergency situations requiring rapid diagnosis and appropriate treatment.

Numerous in vitro assays have been proposed as substitutes for the mouse lethality assay, and these fall into two main categories. The first only detects the neurotoxin protein using specific antibodies (ELISA-based predominantly), or the nucleic acids encoding it (PCR, immuno-PCR). The second includes the methods that assay BoNT/A biological activity (for reviews, Capek and Dickerson, 2010; Hakami et al., 2010; Lindstrom and Korkeala, 2006) .

SPR is a powerful tool for studying biomolecular interactions. The versatility of SPR basedbiosensors extends beyond protein-protein interactions and environmental and medical applications are expanding (Campbell et al., 2011; Helmerhorst et al., 2012). A few studies have used this label-free optical detection technique to measure protease activity using surface-captured substrate (Loew et al., 2000; Shoji et al., 2011; Steinrucke et al., 2000). However in all these reports, protease activity in the fluidic phase was monitored by a loss of substrate mass, leading to a decrease in SPR signal without revealing the site of cleavage.

In this report we describe a new simple and automated method to measure the enzymatic activity of BoNT/A entirely on-chip using SPR. On-chip cleavage of immobilized recombinant SNAP-25 was assayed by detecting neo-epitopes specific to BoNT/A endopeptidase activity. This provides a rapid and sensitive in vitro assay that quantifies BoNT/A activity on-line, by a gain-of-SPR signal.

\section{Material and methods}

2.1 Reagents: Amine coupling kits were from GE Healthcare and Biorad. $\mathrm{ZnCl}_{2}$, Tween 20, dithiothreitol (DTT), Fc specific goat anti-mouse IgG and fatty acid-free bovine serum albumin were from Sigma. Botulinum neurotoxins: frozen aliquots of BoNT/A (Metabiologics, USA) at $3.5 \times 10^{7} \mathrm{LD}_{50} / \mathrm{mg}$ (1 mg/ml from Hall strain) or BoNT/B (Metabiologics, USA) at 1.1 $x 10^{7} \mathrm{LD}_{50} / \mathrm{ml}$ (1 $\mathrm{mg} / \mathrm{ml}$ from Okra strain) were used immediately after thawing. BoNT/E and C (provided by Prof. S Kozaki, Osaka Prefecture University) were produced and purified as previously described (Ferracci et al., 2005). BoNT/A concentrations are indicated as molarity or mouse $\mathrm{LD}_{50} / \mathrm{ml}$. All experiments were performed in accordance with French and European guidelines for handling botulinum neurotoxin. 
2.2 Monoclonal antibody production: Mice were immunized with a synthetic peptide identical to the 191-197 sequence of rat SNAP-25. This sequence is located immediately N-terminal to the peptide bond cleaved by BoNT/A $\left(Q_{197}-R_{198}\right)$. A cysteine residue was added to the $N$ terminus of the peptide to allow coupling to $\mathrm{KLH}$ protein for immunization. Hybridomas were selected by ELISA against the coated peptide and for high affinity binding to SNAP-25 in plasma membrane vesicles treated with BoNT/A (Marconi et al., 2008). Monoclonal antibodies (mAb10F12) were purified by Protein A-Sepharose affinity chromatography and stored at $-20^{\circ} \mathrm{C}$.

2.3 Preparation of SNAP-25 chips: Rat $6 \mathrm{x}$ His-SNAP-25 was cloned in pRSFDuet (Novagen). His-SNAP-25 was expressed in BL21 and purified on Ni-NTA-agarose (Qiagen) beads under denaturing conditions according to the supplier's protocol and dialyzed against HBS (10 mM HEPES-NaOH pH 7.4, $150 \mathrm{mM} \mathrm{NaCl}$ ). Purified proteins were stored in $20 \%$ glycerol at $-80^{\circ} \mathrm{C}$ for a maximum of 6 months. SNAP-25 was amine coupled at $\mathrm{pH} 4.5$ to Biacore CM5 (GE Healthcare) or $\mathrm{pH} 4$ to ProteOn GLH (Biorad) sensor chips according to the manufacturer's instructions using automated programs. The final immobilization density was $9000 \mathrm{RU}(\mathrm{CV}=$ $4 \%, n=15)$ on CM5 chips and $17000 \mathrm{RU}(\mathrm{CV}=3.2 \%, \mathrm{n}=6$ along a channel) on GLH chips, yielding a concentration of 10-30 $\mu \mathrm{M}$. Chips were conditioned by injection of cleavage buffer (50 mM HEPES-NaOH pH 7.4, 0.1\% BSA, 10 mM DTT, 1\% Tween 20, $100 \mu \mathrm{M} \mathrm{ZnCl}_{2}, 10 \mathrm{mM}$ DTT) and background signal was measured from five injections of mAb10F12 diluted in running buffer in the presence of anti-mouse Fc antibody, each at $10 \mu \mathrm{g} / \mathrm{ml}$.

2.4 SPR analysis of BoNT/A endoprotease activity: SPR measurements were performed at $34^{\circ} \mathrm{C}$ using Biacore 3000, Biacore T200 (GE Healthcare) or ProteOn XPR36 (Biorad) apparatuses with TBS (10 mM Tris- $\mathrm{HCl} \mathrm{pH} \mathrm{7.4,} 150 \mathrm{mM} \mathrm{NaCl}$ ) or $\mathrm{HBS}$ as running buffer. The minimum flow rates were $1 \mu \mathrm{l} / \mathrm{min}$ with the Biacore and $25 \mu \mathrm{l} / \mathrm{min}$ with the ProteOn apparatus. BoNT/A samples were serially diluted in cleavage buffer and placed in the sample rack at $4^{\circ} \mathrm{C}$ before injection. In all experiments, cleavage buffer in the absence of BoNT/A was injected onto a control lane coated with SNAP-25 (control flow cell or inter-spot area for Biacore or Biorad apparatus respectively). The pre-assembled mAb10F12/anti-mouse Fc antibody complex was then injected for 2 min over all flow cells. MAb binding was measured $10 \mathrm{sec}$ after the end of injection, and the chip was regenerated with an $8 \mathrm{sec}$ pulse of $10 \mathrm{mM}$ glycine- $\mathrm{HCl} \mathrm{pH}$ 2. The data obtained from the control flow cell were automatically subtracted from experimental measurements to yield the specific signal. The pre-assembled mAb10F12/anti-mouse Fc antibody complex yielded a 3-fold increase in detection sensitivity compared to mAb10F12 alone. Data were analyzed using Biaevaluation 4.1 software (Biacore/GEHeathcare) and ProteOn Manager v. 3.1.0.6 software (Biorad). Assuming a molecular weight ratio of $6: 1$ between mAb and SNAP-25 and that one mAb molecule binds 
to only one epitope at low rates of substrate cleavage, one $R U$ of bound mAb10F12 corresponds to the cleavage of $0.015 \%$ of coupled substrate. The limit of detection (LOD) was measured in the absence of BoNT/A, and defined as the mean of the background plus three times the standard deviation. The limit of quantification (LOQ) was defined as the mean of the background plus ten times the standard deviation. SPR chips were used with at least 40 assay-regeneration cycles without significant loss of binding capacity. Chips were discarded when more than $10 \%$ of the substrate was cleaved.

\section{Results}

3.1 Production of monoclonal antibodies recognizing the reaction product of BoNT/A endopeptidase activity

The proteolytic activity of BoNT/A specifically cleaves a single peptide bond $\left(G \ln _{197}-\operatorname{Arg}_{198}\right)$ of SNAP-25, removing a nine residue (198-206) C-terminal fragment. In order to assay BoNT/A activity using a substrate chip, we produced antibodies designed to bind specifically the Cterminal neo-epitope of SNAP-25 1-197, generated by endopeptidase activity. Mouse monoclonal antibodies were raised against a synthetic peptide corresponding to residues 191-197 of SNAP-25 and screened by ELISA, Western blotting and SPR. Six positive hybridoma clones were obtained, producing mAbs which reacted with SNAP-25 in Western blots of rat brain membrane proteins treated with BoNT/A (Fig. 1a). These mAbs displayed no cross-reactivity with full-length SNAP-25 in untreated membranes. Clone 10F12 was selected for further study on the basis of its high affinity. The specificity of mAb10F12 for SNAP-25 cleaved by BoNT/A versus full length SNAP-25 was also confirmed by SPR experiments using the native protein in membrane fractions (Fig. 1b), as described in (Marconi et al., 2008) and by immunocytochemical staining of cultured neurons incubated in the presence or absence of BoNT/A (Fig. S1).

\subsection{Assay principle}

Biosensors coupled to microfluidics flow cells work on the principle of continuous flow over a target coupled to the sensor surface. Samples are injected into the running buffer, at and for a given time. In this procedure, the target is the BoNT/A substrate, purified recombinant SNAP-25 immobilized on the reaction area called "flow cell" or "spot". The method incorporates two injection steps: (i) a cleavage step in which the sample containing BoNT/A is injected over the substrate. This clips off a nine residue $\mathrm{C}$-terminal peptide and generates a neo-epitope specifically recognized by mAb10F12. (ii) A detection step in which mAb10F12 is injected. MAb10F12 binding to cleaved SNAP-25 produces an SPR signal in real time 
which is directly proportional to the amount of proteolytic product and thus to the concentration of BoNT/A in the initial sample (Fig. 2).

The duration of the sample injection step (ie. enzyme / substrate contact time) was varied between 5 and $300 \mathrm{~min}$ according to the BoNT/A concentration range to be assayed, whereas the detection step was set for 2 minutes. As the SPR signal generated is proportional to the molecular mass of bound molecules, mAb10F12 was pre-mixed with an anti-mouse Fc antibody to increase sensitivity.

\subsection{Assay specificity}

To confirm the specificity of BoNT/A activity detection, other BoNT serotypes were injected over SNAP-25 coated on a Biacore 3000 sensor chip at a relatively high concentrations (1 $\mathrm{nM}$ ). No mAb10F12 binding signal was observed after BoNT/B injection (Fig. 3a), which cleaves the synaptic vesicle protein synaptobrevin / VAMP2. Similarly, BoNT/E and BoNT/C, which cleave SNAP-25 at different sites than BoNT/A (Rossetto et al., 2013), also failed to produce any signal (Fig. 3a). In contrast, injection of BoNT/A at a 1000-fold lower concentration (1 pM) generated a strong signal. These data indicate that the assay is specific for the endoprotease activity of BoNT/A, and qualifies this antibody to identify this serotype in an unknown sample.

\subsection{Cumulative measurements of BoNT/A endoprotease activity}

The SNAP-25 coupling procedure ensures that excess substrate $(>5 \mu \mathrm{M})$ is available. Experiments were systematically carried out either with a low enzyme concentration, or with limited contact time, to ensure that less $<10 \%$ of substrate was cleaved. In these conditions, substrate concentration was considered to be constant during a single or a set of toxin injections in the same flow-cell. Signal repeatability, measured by at least 3 mAb injections, separated by acid regeneration of the sensor chip, was high (CV 1\% from 4 to $5000 \mathrm{RU}$, and $4 \%$ for $2 \mathrm{RU})$. Hence in principle several measurements can be performed in the same flow cell by monitoring successive increments in signal. Fig. 3b illustrates 7 consecutive injections of the same toxin concentration for $5 \mathrm{~min}$, followed in each case by an independent mAb10F12 injection. Product formation over each $5 \mathrm{~min}$ period proceeds at a linear rate indicating that the reaction is not substrate-limited (Fig. 3c). At a fixed reference time this rate will depend linearly on enzyme concentration (see below).

Endoprotease activity were found to be independent of the buffer flow rate over the sensorchip between the limits of 1 and $25 \mu \mathrm{l} / \mathrm{min}$ (Fig. S2), indicating that diffusional transport of the enzyme to the solid-state substrate is non-limiting compared to the enzymatic reaction. 


\section{5: Rapid detection of BoNT/A activity}

The rapidity of BoNT/A detection is of major importance in surveillance applications, thus the potential of SPR to achieve this aim was evaluated at $1 \mathrm{LD}_{50} / \mathrm{ml}$, a concentration that corresponds to the limit of detection for the in vivo mouse assay obtained in $24 \mathrm{~h}$. In order to improve measurement sensitivity, experiments were carried out on a Biacore T200 apparatus which provides very low background. As illustrated in Fig. 4, specific endoprotease activity was readily detected after a 5 min injection of reduced BoNT/A, yielding $4.5 \pm 1 R U(n=3)$, a value largely above the LOD (dashed line). Alternatively, non-reduced BoNT/A was diluted in cleavage buffer containing DTT and immediately injected. In these conditions, although all BoNT/A was not fully reduced and thus active, a cleavage time of 15 min was sufficient to clearly detect BoNT/A activity yielding $10.7 \pm 1 \mathrm{RU}(\mathrm{n}=4$, data not shown). These data indicate that our procedure provides a sensitivity of $1 \mathrm{LD}_{50} / \mathrm{ml}(55 \mathrm{fM}$ BoNT/A, i.e $1.3 \mathrm{pg}$ of BoNT/A) within a time scale of 5-15 minutes injection.

\subsection{Time-dependent sensitivity of the assay:}

The dose-dependency of the method was evaluated using prolonged cleavage time with the multispot ProteOn biosensor. This device has a slightly lower sensitivity than the Biacore apparatus but has the advantage of allowing simultaneous injection of 6 samples over 6 different substrate-coated spots. This characteristic allows a direct comparison of SPR signals produced by different BoNT/A concentrations and dramatically increases the speed of sample analysis. Concentrations of BoNT/A ranging between 0.1 and $3 L_{50} / \mathrm{ml}$ were injected 4 times each for a 15 min duration. Time course and dose-response curves were linear (Fig. S3 and Fig. 5a). $1 \mathrm{LD}_{50} / \mathrm{ml}$ was detected in $15 \mathrm{~min}$ (Fig. 5a) and $0.1 \mathrm{LD}_{50} / \mathrm{ml}$ in 1 hour (Fig. 5b). A linear dose-response relationship was also measured when BoNT/A (0.01 - $0.3 \mathrm{LD}_{50} / \mathrm{ml}$ ) was injected for $5 \mathrm{~h}$ (Fig. $5 \mathrm{c}$ ). In this case the LOD attained $0.01 \mathrm{LD}_{50} / \mathrm{ml}$ (Fig. $5 \mathrm{~d})$ and the LOQ was $0.03 \mathrm{LD}_{50} / \mathrm{ml}$. Using a Biacore T200 the LOD was $0.03 \mathrm{LD}_{50} / \mathrm{ml}(1.5 \mathrm{fM})$ and $0.008 \mathrm{LD}_{50} / \mathrm{ml}$ (400 attomolar; $1 \mathrm{aM}=10^{-18} \mathrm{M}$ ) for 1 and 5 hour injections respectively (data not shown).

\section{Discussion}

We have developed an SPR method to detect and assay the activity of botulinum neurotoxin $A$, one of the most potent neurotoxins in humans. This fully automated assay consists in injecting samples containing BoNT/A over a chip coated with substrate that can then be rapidly probed to measure the enzymatic product. BoNT/A cleaves a specific peptide bond, removing a six amino acid fragment and generating a new C-terminus. Production of a 
custom mAb directed against the C-terminal neo-epitope was thus crucial, as the specificity of the probe/analyte interaction determines an effective assay. Only two biological reagents, substrate and mAb10F12 are required to perform the assay and samples are directly analyzed with minimal handling.

This assay is versatile as the contact time between BoNT/A and substrate can be modified depending on the purpose of the assay and the enzyme concentration to be determined. 1 $\mathrm{LD}_{50} / \mathrm{ml}(55 \mathrm{fM})$ was detected within a few minutes, in contrast to several hours required for the in vivo mouse assay. For applications in which extremely low concentrations of BoNT/A were to be detected, prolonging the cleavage time achieved a sensitivity of $0.008 \mathrm{LD}_{50} / \mathrm{ml}$ $(400 \mathrm{aM})$ in $5 \mathrm{~h}$. We predict that longer injection periods could attain even higher sensitivity in the attomolar range. A biosensor able to process simultaneous injections was used to generate linear dose response-curves for various contact times, demonstrating the potential of the method for quantitation down to $0.03 L_{50} / \mathrm{ml}$.

Among the fastest tests for the detection of BoNT are those based on direct fluorescence measurement associated with substrate cleavage in solution. Although these assays are very appropriate for automated high-throughput screening of BoNT/A inhibitors, they lack sensitivity compared to the SPR assay: $1 \mathrm{nM}$ BoNT/A detected in 2 minutes (Schmidt and Stafford, 2003), 4 pM detected in 120 min (Poras et al., 2009) versus $55 \mathrm{fM}$ in 5 minutes for our assay. Although the SPR assay includes two steps: cleavage and detection, it can still compete with single-step assays in terms of rapidity, because detection is monitored on-line in the first few seconds, by a gain-of signal resulting from BoNT/A action.

The SPR procedure attains sensitivity similar to an optimized ELISA assay which constitutes to date the most sensitive enzymatic assay reported without prior concentration of BoNT/A (Ekong et al., 1997; Jones et al., 2007). However, ELISA is a multistep, labor-intensive technique that requires long incubation periods. The automated SPR method offers several advantages over ELISA including shorter cleavage time ( $5 \mathrm{~h}$ versus $18 \mathrm{~h}$ ) and detection time (minutes versus hours).

Several factors could contribute to the high sensitivity of the assay. Substrate immobilization on negatively charged hydrogels (dextran or alginate), that maintain SNAP-25 in an accessible conformation might accelerate enzymatic cleavage. Furthermore, as BoNT/A is positively charged at $\mathrm{pH} 7.4$, it is possible that the negatively charged hydrogel concentrates the toxin and promotes its enzymatic activity in a similar way to negatively charged liposomes (Caccin et al., 2003). This kind of effect has been previously described for alphachymotrypsin acting on immobilized substrate (Deere et al., 2008). 
No loss of mass was detected during protease injection. This is due to the fact that only a small percentage of the substrate was cleaved and furthermore the released peptide (9 amino acids) accounts for only $4 \%$ of the total mass of SNAP-25. This procedure has several advantages over biosensor assays based on the measurement of mass loss upon enzymatic cleavage. Specific monitoring of the reaction product avoids false positives resulting from the action of other BoNT serotypes or non-specific proteolysis. Moreover it does not require coupling to other methods (eg. mass spectrometry) to analyze reaction products (Henn et al.,

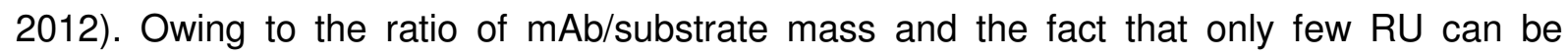
accurately measured, the method can in theory detect enzymatic cleavage of as low as $0.005 \%$ of the total immobilized substrate. This precision cannot be achieved by detecting loss of mass. Measurement of a minute amount of cleaved substrate authorizes multiple reuse of the sensor allowing us to monitor cleavage kinetics rather than a single end-point measurement or to repeat multiple assays on the same flow cell compatible with inhibitorscreening applications.

\section{Conclusions}

In conclusion this new biosensor method provides a simple, rapid and highly sensitive in vitro assay for the enzymatic activity of BoNT/A. SPR biosensors can operate in various media (Ferracci et al., 2011) and the presence of BoNT/A could be detected in liquid foods using the method proposed. Alternatively the biosensor could be directly connected to a supply of drinking water and operate automatically to continuously monitor the presence of BoNT/A by repeatedly injecting the specific $m A b$ at fixed times. In the future the method could be extended to detect all BoNTs that affect humans, providing antibodies that specifically recognize cleaved-substrates can be generated.

ACKNOWLEDGMENTS: This work was supported by a grant from the DGA (REI $N^{\circ}$ 0634040) 


\section{LEGENDS TO FIGURES}

Fig. 1: Characterization of monoclonal antibodies designed to recognize the product of SNAP-25 cleavage by botulinum neurotoxin $A$.

a) A rat brain membrane fraction (Marconi et al., 2008) was incubated in the presence or absence of $10 \mathrm{nM}$ BoNT/A. Western blots of membranes were stained with $5 \mu \mathrm{g} / \mathrm{ml}$ of six mAbs raised against the 191-197 peptide of SNAP-25. In all cases the major immunoreactive bands correspond to the 1-197 fragment of SNAP-25 generated by BoNT/A endoprotease activity. Immunoreactivity was not detected in samples untreated by BoNT/A.

b) mAb10F12 was immobilized on the active flow cell of a Biacore 3000 apparatus and an irrelevant $\mathrm{mAb}$ on a control flow cell. Plasma membrane-derived vesicles (Marconi et al. 2008) pre-treated with BoNT/A (10 nM) were injected in the absence (upper trace) or presence (lower trace) of an excess of SNAP-25 191-197 peptide (1 $\mu \mathrm{M})$.

Fig. 2: Assay for the endoprotease activity of botulinum neurotoxin A: general principle.

A sample containing BoNT/A was injected $300 \mathrm{sec}$ over the SNAP-25 sensorchip. The deflection to approximately $600 \mathrm{RU}$ and complete return to baseline reflects the change in refractive index during sample injection, irrespective of the presence (red trace) or absence (black trace) of BoNT/A (400 fM). MAb10F12 was then injected to detect epitopes generated by BoNT/A activity. The difference between red and black traces) corresponds to specific $\mathrm{mAb}$ binding, and indicates the number of neo-epitopes produced, which is directly related to BoNT/A activity. Each trace represents a sensorgram monitored in the single flow-cell of a Biacore sensor chip.

Fig. 3: Botulinum neurotoxin A: assay specificity and kinetics.

a) Samples containing BoNT/A (1 pM), BoNT/B, BoNT/C, BoNT/E (all at $1 \mathrm{nM}$ ) or without BoNT (Cont.) were diluted in cleavage buffer and injected for 10 min over a SNAP-25 sensorchip. SNAP-25 cleavage was detected by injection of mAb10F12 $( \pm S D, n=3$ mAb injections). Results are representative of 3 independent experiments. b) Seven successive injections of BoNT/A (200 fM) over immobilized SNAP-25 for a duration of $5 \mathrm{~min}$. After each injection of toxin, mAb10F12 was injected and the amount of cleaved SNAP-25 measured 10 sec after the end of injection plotted in c). 
Fig. 4: Rapid SPR detection of botulinum neurotoxin A at the mouse bioassay threshold.

Cleavage buffer in the presence or absence of $55 \mathrm{fM} \mathrm{BoNT/A}\left(1 \mathrm{LD}_{50} / \mathrm{ml}\right)$ was injected over a SNAP-25 sensorchip (Biacore T200) for 5 minutes and cleavage products immediately detected using mAb10F12. Traces represent a typical experiment with superimposed signals generated by 3 injections of mAb10F12. Experiments in the absence of BoNT/A yielded a mean of $-0.7 \pm 0.4 \mathrm{RU}(\mathrm{n}=7$ independent experiments) and a LOD of $0.5 \mathrm{RU}$ (dashed line).

Fig. 5: Multispot SPR analysis of botulinum neurotoxin A activity.

(a) Six samples containing the indicated concentrations of BoNT/A were injected in parallel for $15 \mathrm{~min}$ and then cleaved SNAP-25 was measured from mAb10F12 binding $(n=3)$. This procedure was then repeated 3 times to reach a cleavage time of $60 \mathrm{~min}$. (b) Three superimposed sensorgrams at $0.1,0.3$ and $1 \mathrm{LD}_{50} / \mathrm{ml}$ BoNT/A at the final 60 min time point in (a). (c) as in (a) with a lower BoNT/A concentration range and a single $5 \mathrm{~h}$ injection. (d) as in (b) with a lower BoNT/A concentration range and a single $5 \mathrm{~h}$ injection. Dashed lines in (a) and (c) denote the mean background level from 3 independent experiments plus 3 SD. The figure is representative of 3 independent experiments (error bars which are often smaller than data points represent SD calculated from $3 \mathrm{mAb}$ injections).

\section{Supplemental Data Legends}

Fig. S1: Detection of SNAP-25 cleaved by botulinum neurotoxin $A$ in cultured neurons.

Cultured rat hippocampal neurons were prepared as described (Ferracci et al., 2011). Cells at 15 days in vitro were either untreated (control) or exposed to BoNT/A (1 nM) for 3 hours. Cells were then fixed for immunochemistry. SNAP-25 cleavage was detected using mAb10F12. Asterisk indicates soma localization. Scale bar $=20 \mu \mathrm{m}$.

Fig. S2: Influence of flow rate on protease activity.

BoNT/A (100 fM) was injected over surface-bound SNAP-25 (Biacore 3000) for 5 minutes at flow rates of 1,5 or $25 \mu \mathrm{l} / \mathrm{ml}$, followed by injection of mAb10F12. Representative of 3 independent experiments. 


\section{Fig. S3: Kinetics of botulinum neurotoxin A using a multispot biosensor.}

Six samples containing the indicated concentrations of BoNT/A were injected for 15 min and then cleaved SNAP-25 was measured from mAb10F12 binding $(n=3)$. This procedure was then repeated 3 times at each concentration, ie. a total of 4 BoNT/A injections over 1 hour. Error bars are not visible. Representative of 3 independent experiments.

\section{References}

Arnon, S.S., Schechter, R., Inglesby, T.V., Henderson, D.A., Bartlett, J.G., Ascher, M.S., Eitzen, E., Fine, A.D., Hauer, J., Layton, M., Lillibridge, S., Osterholm, M.T., O'Toole, T., Parker, G., Perl, T.M., Russell, P.K., Swerdlow, D.L., Tonat, K., 2001. Botulinum toxin as a biological weapon: medical and public health management. Jama 285(8), 1059-1070.

Caccin, P., Rossetto, O., Rigoni, M., Johnson, E., Schiavo, G., Montecucco, C., 2003. VAMP/synaptobrevin cleavage by tetanus and botulinum neurotoxins is strongly enhanced by acidic liposomes. FEBS letters 542(1-3), 132-136.

Campbell, K., McGrath, T., Sjolander, S., Hanson, T., Tidare, M., Jansson, O., Moberg, A., Mooney, M., Elliott, C., Buijs, J., 2011. Use of a novel micro-fluidic device to create arrays for multiplex analysis of large and small molecular weight compounds by surface plasmon resonance. Biosensors \& bioelectronics 26(6), 3029-3036.

Capek, P., Dickerson, T.J., 2010. Sensing the deadliest toxin: technologies for botulinum neurotoxin detection. Toxins 2(1), 24-53.

Deere, J., De Oliveira, R.F., Tomaszewski, B., Millar, S., Lalaouni, A., Solares, L.F., Flitsch, S.L., Halling, P.J., 2008. Kinetics of enzyme attack on substrates covalently attached to solid surfaces: influence of spacer chain length, immobilized substrate surface concentration and surface charge. Langmuir 24(20), 11762-11769.

Dhaked, R.K., Singh, M.K., Singh, P., Gupta, P., 2010. Botulinum toxin: bioweapon \& magic drug. The Indian journal of medical research 132, 489-503. 
Ekong, T.A., Feavers, I.M., Sesardic, D., 1997. Recombinant SNAP-25 is an effective substrate for Clostridium botulinum type A toxin endopeptidase activity in vitro. Microbiology (Reading, England) 143 ( Pt 10), 3337-3347.

Ferracci, G., Marconi, S., Mazuet, C., Jover, E., Blanchard, M.P., Seagar, M., Popoff, M., Leveque, C., 2011. A label-free biosensor assay for botulinum neurotoxin B in food and human serum. Analytical biochemistry 410(2), 281-288.

Ferracci, G., Miquelis, R., Kozaki, S., Seagar, M., Leveque, C., 2005. Synaptic vesicle chips to assay botulinum neurotoxins. The Biochemical journal 391(Pt 3), 659-666.

Hakami, R.M., Ruthel, G., Stahl, A.M., Bavari, S., 2010. Gaining ground: assays for therapeutics against botulinum neurotoxin. Trends in microbiology 18(4), 164-172.

Helmerhorst, E., Chandler, D.J., Nussio, M., Mamotte, C.D., 2012. Real-time and Label-free Bio-sensing of Molecular Interactions by Surface Plasmon Resonance: A Laboratory Medicine Perspective. The Clinical biochemist 33(4), 161-173.

Henn, C., Boettcher, S., Steinbach, A., Hartmann, R.W., 2012. Catalytic enzyme activity on a biosensor chip: combination of surface plasmon resonance and mass spectrometry. Analytical biochemistry 428(1), 28-30.

Jones, O.M., 2007. The antinociceptive effects of botulinum toxin therapy for anal fissure are unproven. Diseases of the colon and rectum 50(5), 682-683; author reply 683.

Lindstrom, M., Korkeala, H., 2006. Laboratory diagnostics of botulism. Clinical microbiology reviews 19(2), 298-314.

Loew, D., Perrault, C., Morales, M., Moog, S., Ravanat, C., Schuhler, S., Arcone, R., Pietropaolo, C., Cazenave, J.P., van Dorsselaer, A., Lanza, F., 2000. Proteolysis of the exodomain of recombinant protease-activated receptors: prediction of receptor activation or inactivation by MALDI mass spectrometry. Biochemistry 39(35), 10812-10822.

Marconi, S., Ferracci, G., Berthomieu, M., Kozaki, S., Miquelis, R., Boucraut, J., Seagar, M., Leveque, C., 2008. A protein chip membrane-capture assay for botulinum neurotoxin activity. Toxicology and applied pharmacology 233(3), 439-446. 
Poras, H., Ouimet, T., Orng, S.V., Fournie-Zaluski, M.C., Popoff, M.R., Roques, B.P., 2009. Detection and quantification of botulinum neurotoxin type a by a novel rapid in vitro fluorimetric assay. Applied and environmental microbiology 75(13), 4382-4390.

Rossetto, O., Megighian, A., Scorzeto, M., Montecucco, C., 2013. Botulinum neurotoxins. Toxicon 67C, 31-36.

Schmidt, J.J., Stafford, R.G., 2003. Fluorigenic substrates for the protease activities of botulinum neurotoxins, serotypes A, B, and F. Applied and environmental microbiology 69(1), 297-303.

Shoji, A., Kabeya, M., Sugawara, M., 2011. Real-time monitoring of matrix metalloproteinase- 9 collagenolytic activity with a surface plasmon resonance biosensor. Analytical biochemistry 419(1), 53-60.

Steinrucke, P., Aldinger, U., Hill, O., Hillisch, A., Basch, R., Diekmann, S., 2000. Design of helical proteins for real-time endoprotease assays. Analytical biochemistry 286(1), 26-34. 